\title{
On-chip Q-factor greater than 1 billion
}

\author{
Lue Wu ${ }^{1, \dagger}$, Heming Wang ${ }^{1, \dagger}$, Qi-Fan Yang ${ }^{1, \dagger}$, Maodong Gao ${ }^{1}$, Qing-Xin Ji ${ }^{1,2}$, \\ Boqiang Shen ${ }^{1}$, Chengying Bao ${ }^{1}$, and Kerry Vahala ${ }^{1, *}$ \\ ${ }^{1}$ T. J. Watson Laboratory of Applied Physics, California Institute of Technology, Pasadena, CA 91125, USA \\ ${ }^{2}$ School of Physics, Peking University, Beijing 100871, China \\ *vahala@caltech.edu
}

\begin{abstract}
A record Q-factor of 1.1 billion is demonstrated in on-chip silica whisperinggallery resonators. Using the devices, sub-milliwatt parametric oscillation threshold is measured in $9 \mathrm{GHz}$ free-spectral-range devices. (C) 2020 The Author(s)
\end{abstract}

OCIS codes: (190.4360) Nonlinear optics, devices; (140.3945) Microcavities

Optical microresonators with ultra-high quality factors (Q-factors) are ideal platforms for study of a wide range of phenomena including nonlinear optics and cavity quantum electrodynamics [1]. They are also powerful tools for applications including spectrometers, ranging systems, ultra-stable microwave sources and gyroscopes. Here, by employing optimized fabrication methods, a record on-chip $Q$ factor of 1.1 billion is demonstrated for wedge resonators [2]. As an additional verification of the $\mathrm{Q}$ factor, sub-milliwatt parametric oscillation threshold is demonstrated at the challenging free-spectral-range (FSR) of $9 \mathrm{GHz}$.

Devices are fabricated using a combination of lithography and wet and dry etching. Intrinsic $Q$ factors are measured by characterizing resonance linewidths (accounting for loading effects) and through cavity ring down measurements. Fig. 1a plots the highest intrinsic Q-factors $Q_{0}$ obtained at device diameters ranging from $3 \mathrm{~mm}$ to $7.2 \mathrm{~mm}$. The near-linear dependence of $Q_{0}$ on diameter (red dashed line) indicates an approximately constant finesse according to $Q / D=n \pi / \lambda_{\text {res }} \times F$, where $F$ is finesse, $n$ is refractive index and $\lambda_{\text {res }}$ is resonance wavelength.

Using a device having an FSR of approximately $10 \mathrm{GHz}$, the full-width-at-half-maximum (FWHM) linewidth is measured (at $1585 \mathrm{~nm}$ ) to be $220 \mathrm{kHz}$, corresponding to a loaded Q factor $Q_{L}=860 \mathrm{M}$. By measuring the
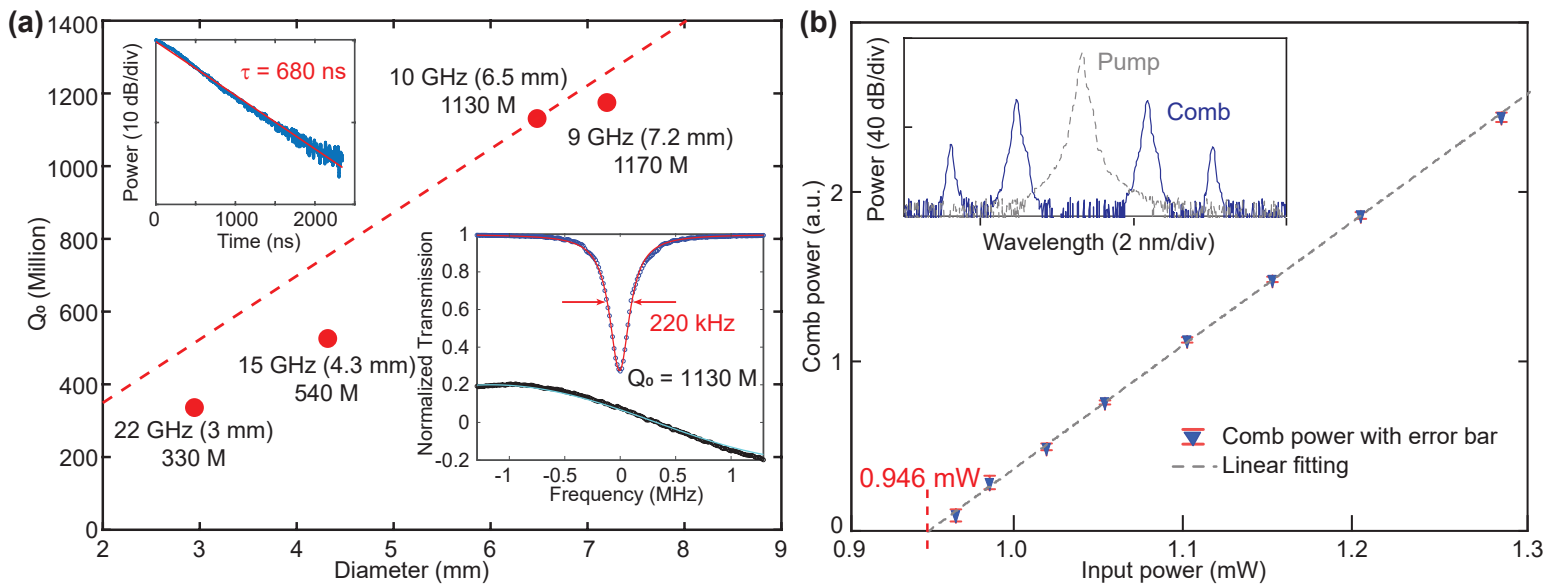

Fig. 1. (a) $Q_{0}$ data for microresonators with varying size. Red dashed line corresponds to a finesse of 60,000. Right inset: Resonance linewidth measurement of a $10 \mathrm{GHz}$ device at $1585 \mathrm{~nm}$. Top trace shows measured resonance transmission (blue dots) with Lorentzian lineshape fitting (red curve). Bottom trace shows measured output of a Mach-Zehnder interferometer (black dots) with sinusoidal fitting (cyan curve) to calibrate the frequency (the FSR of the interferometer is $5.979 \mathrm{MHz}$ ). The linewidth is fitted to be $220 \mathrm{kHz}$, corresponding to intrinsic $Q_{0}=1.1$ billion and loaded $Q_{L}=860$ M. Left inset: Ring down measurement of the same device. Measured output power (blue) is fitted with an exponential decay function (red), giving fitted photon lifetime of $680 \mathrm{ns,} \mathrm{corresponding} \mathrm{to}$ loaded $Q_{L} \approx 810 \mathrm{M}$. (b) Plot of parametric oscillation power versus input pump power for a $9 \mathrm{GHz}$ device at $1550 \mathrm{~nm}$. Linear fitting gives parametric oscillation threshold of $0.946 \mathrm{~mW}$. Inset: Optical spectrum of pump (dashed gray) and parametric oscillation comb (solid blue) with $1.12 \mathrm{~mW}$ input power. 
transmission depth, a coupling Q factor of $Q_{1}=3.6$ billion is determined from which an intrinsic $Q_{0}=1.1$ billion is inferred. For the same device, the fitting of ring-down of intracavity power gives a photon lifetime of about $680 \mathrm{~ns}$, which corresponds to a loaded $Q$ factor of approximately 810 million. The linewidth and ring-down data agree well with each other. To the authors' knowledge these are the highest optical Q factors reported for on-chip devices.

To further confirm the Q measurements, parametric oscillation threshold is measured. As shown in Fig. 1b, sub-milliwatt on-chip oscillation threshold $(0.946 \mathrm{~mW})$ is achieved using a $9 \mathrm{GHz}$ FSR device at $1550 \mathrm{~nm}$. This is a record low threshold for this low FSR [3]. The comb spectrum just above the threshold (1.12 $\mathrm{mW}$ input power) is presented in the inset. For the measurement, the pump was nearly completely filtered to obtain the threshold plot. It has been inserted in the parametric oscillation comb spectrum as the dashed curve in the inset.

In summary, we have demonstrated a record on-chip Q-factor of 1.1 billion in silica optical resonators. The $Q$ values were also verified by measurement of the parametric oscillation threshold, which is also a record low value at the FSR value of $9 \mathrm{GHz}$. The resulting improvement in Q will benefit low-power applications for soliton microcombs [4,5] and Brillouin lasers [6].

This work is supported by DARPA (HR0011-15-C-055), AFOSR (FA9550-18-1-0353) and the Kavli Nanoscience Institute.

$\dagger$ These authors contributed equally to this work.

\section{References}

1. K. J. Vahala, "Optical microcavities," Nature 424, 839-846 (2003).

2. H. Lee, T. Chen, J. Li, K. Y. Yang, S. Jeon, O. Painter, and K. J. Vahala, "Chemically etched ultrahigh-Q wedge-resonator on a silicon chip," Nat. Photon. 6, 369-373 (2012).

3. M.-G. Suh and K. Vahala, "Gigahertz-repetition-rate soliton microcombs," Optica 5, 65-66 (2018).

4. T. J. Kippenberg, A. L. Gaeta, M. Lipson, and M. L. Gorodetsky, "Dissipative kerr solitons in optical microresonators," Science 361 (2018).

5. X. Yi, Q.-F. Yang, K. Y. Yang, M.-G. Suh, and K. Vahala, "Soliton frequency comb at microwave rates in a high-q silica microresonator," Optica 2, 1078-1085 (2015).

6. J. Li, H. Lee, T. Chen, and K. J. Vahala, "Characterization of a high coherence, brillouin microcavity laser on silicon,” Opt. express 20, 20170-20180 (2012). 